\title{
Left-Right Symmetry and Leading Contributions to Neutrinoless Double Beta Decay
}

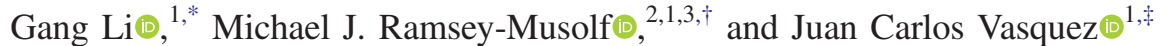 \\ ${ }^{1}$ Amherst Center for Fundamental Interactions, Department of Physics, University of Massachusetts, \\ Amherst, Massachusetts 01003, USA \\ ${ }^{2}$ Tsung-Dao Lee Institute and School of Physics and Astronomy, Shanghai Jiao Tong University, \\ 800 Dongchuan Road, Shanghai 200240, China \\ ${ }^{3}$ Kellogg Radiation Laboratory, California Institute of Technology, Pasadena, California 91125, USA
}

(Received 21 October 2020; accepted 8 March 2021; published 16 April 2021)

\begin{abstract}
We study the impact of the mixing (LR mixing) between the standard model $W$ boson and its hypothetical, heavier right-handed parter $W_{R}$ on the neutrinoless double beta decay $(0 \nu \beta \beta$ decay) rate. Our study is done in the minimal left-right symmetric model assuming a type-II dominance scenario with charge conjugation as the left-right symmetry. We then show that the $0 \nu \beta \beta$ decay rate may be dominated by the contribution proportional to this LR mixing, which at the hadronic level induces the leading-order contribution to the interaction between two pions and two charged leptons. The resulting long-range pion exchange contribution can significantly enhance the decay rate compared to previously considered shortrange contributions. Finally, we find that even if future cosmological experiments rule out the inverted hierarchy for neutrino masses, there are still good prospects for a positive signal in the next generation of $0 \nu \beta \beta$ decay experiments.
\end{abstract}

DOI: 10.1103/PhysRevLett.126.151801

Determining the properties of the light neutrinos under charge conjugation is a key challenge for particle and nuclear physics. As the only electrically neutral fermions in the standard model (SM) of particle physics, neutrinos are the sole SM candidates for possessing a Majorana mass. The corresponding term in the Lagrangian breaks the conservation of total lepton number $(L)$ by two units: $\mathcal{L}_{M} \supset-y_{\nu} \overline{\ell^{C}} H^{T} H \ell / \Lambda$, where $\ell$ and $H$ are the SM lefthanded lepton doublet and Higgs doublet, respectively, and $\Lambda$ is a mass scale whose presence is needed to maintain dimensionality. After the neutral component of the Higgs doublet obtains a vacuum expectation value $v / \sqrt{2}$, the resulting Majorana mass operator is $\mathcal{L}_{M} \rightarrow-\left(m_{\nu} / 2\right) \overline{\nu^{c}} \nu$, with $m_{\nu}=y_{\nu} v^{2} / \Lambda$. For $y_{\nu} \sim \mathcal{O}(1)$, the observed scale of light neutrino masses consistent with oscillation experiments [1] and cosmological bounds [2,3] would imply $\Lambda \gtrsim 10^{15} \mathrm{GeV}$.

An experimental determination that neutrinos are Majorana fermions could, thus, provide circumstantial evidence for $L$-violating processes at ultrahigh-energy scales involving new particles not directly accessible in the laboratory. In the widely considered seesaw mechanism, the $L$-violating, out-of-equilibrium decays of these particles

Published by the American Physical Society under the terms of the Creative Commons Attribution 4.0 International license. Further distribution of this work must maintain attribution to the author(s) and the published article's title, journal citation, and DOI. Funded by SCOAP ${ }^{3}$. (fermions) could generate the cosmic matter-antimatter asymmetry [4]. Neutrino oscillation experiments are agnostic regarding the existence of a Majorana neutrino mass term. However, the observation of $0 \nu \beta \beta$ decay in the nuclear transition $(A, Z) \rightarrow(A, Z+2)+e^{-}+e^{-}[5]-a$ process that also violates $L$ by two units-would provide conclusive evidence that light neutrinos are Majorana fermions [6].

The recent $0 \nu \beta \beta$ decay search in the KamLAND-Zen experiment [7] provides the most stringent upper limit on the effective Majorana mass $\left|m_{\beta \beta}\right|$, which is 0.061$0.165 \mathrm{eV}$ at $90 \%$ confidence level (C.L.), where the range reflects the uncertainty in nuclear matrix element computations. In the three-neutrino framework [8], $\left|m_{\beta \beta}\right|$ depends on the neutrino mass spectrum. In the inverted hierarchy (IH) it is bounded below $\left|m_{\beta \beta}\right| \gtrsim 0.01 \mathrm{eV}$, while in the normal hierarchy $(\mathrm{NH})$ it can be vanishingly small. The next generation of $0 \nu \beta \beta$ decay searches with ton-scale detectors [9-14] aim for sensitivities for $\left|m_{\beta \beta}\right|$ as low as $0.01 \mathrm{eV}$. If neutrinos are Majorana fermions, and if the $\mathrm{IH}$ is realized in nature, one would thus expect a nonzero result in the ton-scale experiments.

Cosmological observations provide complementary information on neutrino masses, currently constraining the sum of neutrino masses (dubbed $\Sigma m_{\nu}$ ) to be smaller than $0.12 \mathrm{eV}$ at the $2 \sigma$ level [15]. Global fits [2,3] of neutrino oscillation data, $0 \nu \beta \beta$ decay search results, and cosmological surveys show that the $\mathrm{NH}$ is favored over the IH at about $2 \sigma$ level. For future cosmological surveys [16-20], it is possible to exclude the $\mathrm{IH}$, while the favored $\left|m_{\beta \beta}\right|$ may be out of the reach of ton-scale $0 \nu \beta \beta$ 
decay experiments [9-14] in the three-neutrino framework. Then, it is natural to ask how one could interpret a $0 \nu \beta \beta$ decay signal if cosmological measurements and/or future oscillation experiments demonstrate conclusively that the light neutrino mass ordering is in the $\mathrm{NH}$.

Here, we address this question in the context of one of the most extensively studied extensions of the SM that generically implies the existence of Majorana neutrinos: the minimal left-right symmetric model (LRSM) [21-26]. This model may have $\mathrm{TeV}$ scale new particles and the contributions to the $0 \nu \beta \beta$ decay from the new right-handed sector can be appreciable. The light neutrino and new physics contributions are characterized by $G_{F}^{2}\left|m_{\beta \beta}\right| / p^{2}$ and $c / \Lambda^{5}$ [27-30], respectively. Here, the virtual neutrino momentum $p \simeq 100 \mathrm{MeV}, G_{F}$ is the Fermi constant, and $c$ denotes new Yukawa and/or gauge couplings. For $c \simeq \mathcal{O}(1)$ and $\left|m_{\beta \beta}\right| \simeq 0.1(0.01) \mathrm{eV}$, the new physics contribution can be comparable to the light neutrino contribution if $\Lambda \simeq 3.7(5.9) \mathrm{TeV}$. In particular, it has been shown $[31,32]$ that in the minimal LRSM the contributions coming from heavy neutrinos from the exchange of two righthanded $W_{R}$ bosons (the RR amplitude), see Fig. 1(a), are sizable. Nonetheless, the bulk of the minimal LRSM parameter space would remain largely inaccessible to ton-scale $0 \nu \beta \beta$ decay searches if cosmological data push the bound on $\Sigma m_{\nu}$ below $\sim 0.1 \mathrm{eV}$.

In what follows, we show that this conclusion changes dramatically in the presence of mixing between the left- and

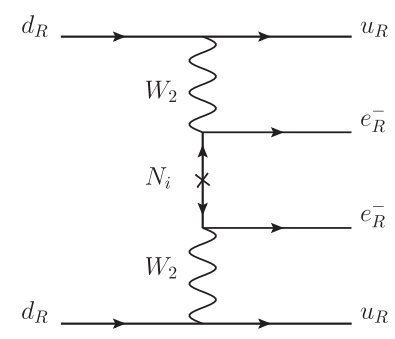

(a)

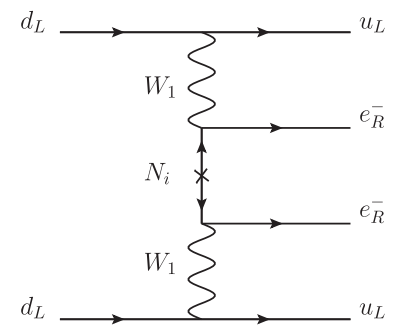

(c)

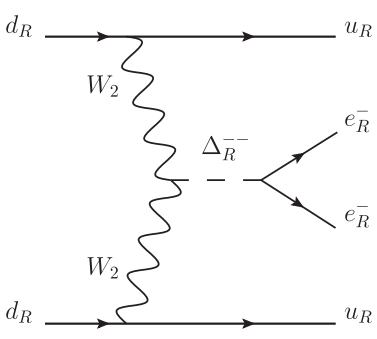

(b)

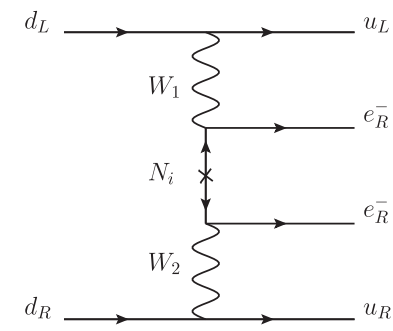

(d)
FIG. 1. Feynman diagrams in the minimal LRSM contributing to the $0 \nu \beta \beta$ decay. Panels (a) corresponds to the RR amplitude, panel (b) denotes the contribution from the exchange of the doubly charged scalar, panel (c) denotes the contribution from the exchange of the SM $W$ boson and heavy neutrinos, while panel (d) corresponds to the LR amplitude. right-handed gauge bosons. This mixing results in contributions to the decay amplitude involving the exchange of heavy right-handed neutrinos, one SM $W$ boson (predominantly left-handed) and one heavy $W$ boson (predominantly right-handed) - a contribution we denote as the LR amplitude; see Fig. 1(d). In Refs. [33,34] it was found that the LR amplitude is suppressed with respect to the RR amplitude due to the upper bounds on the $W_{L}-W_{R}$ mixing angle. However, those studies did not include long-range contributions associated with pion exchange that significantly enhance LR amplitude and can compensate for this suppression [35]. It is also worthwhile to stress that the new contributions due to the left-right mixing as depicted in Fig. 1(d) are different from those given by the so-called $\lambda$ and $\eta$ diagrams considered in Refs. [32,34]. In this Letter, we compute these long-range contributions using state-ofthe-art information on hadronic and nuclear matrix elements as well as phenomenological constraints on the relevant minimal LRSM parameters. We find that even in the presence of prospective, stringent cosmological bounds on $\Sigma m_{\nu}$ and possible exclusion of the IH, there exists ample opportunity for the observation of a signal in next generation $0 \nu \beta \beta$ decay searches.

This framework entails extending the SM gauge group to $\mathrm{SU}(3)_{C} \times \mathrm{SU}(2)_{L} \times \mathrm{SU}(2)_{R} \times \mathrm{U}(1)_{B-L}$, where $B$ and $L$ denote the SM Abelian baryon and lepton quantum numbers. The Higgs sector consists of two scalar triplets $\Delta_{L} \in(1,3,2), \quad \Delta_{R} \in(3,1,2)$ and one bidoublet $\Phi \in(2,2,0)$, where $(X, Y, Z)$ denote the representations under the $\mathrm{SU}(2)_{R, L}$ and $\mathrm{U}(1)_{B-L}$ groups. The neutral components of the bidoublet field $\Phi$ obtain vacuum expectation values: $\langle\Phi\rangle \rightarrow \operatorname{diag}\left\{v_{1}, v_{2} e^{i \alpha}\right\}$, with $v=$ $\sqrt{v_{1}^{2}+v_{2}^{2}}$ and $\alpha$ being the spontaneous $C P$-violating phase.

Of particular relevance to $0 \nu \beta \beta$ decay is the chargedcurrent Lagrangian,

$$
\begin{aligned}
\mathcal{L}_{\mathrm{CC}}= & -\frac{g}{\sqrt{2}} \sum_{A=L, R}\left\{\bar{u}_{A i} V_{A i j}^{\mathrm{CKM}} W_{A} d_{A j}\right. \\
& \left.-\bar{e}_{A i} V_{A i j} W_{A} \nu_{A j}\right\}+ \text { H.c. },
\end{aligned}
$$

where $A=L, R$ and $V_{L, R}^{\mathrm{CKM}}$ and $V_{L, R}$ are the CabiboKobayashi-Maskawa (CKM) and lepton-mixing matrices, respectively. The $L, R$ gauge bosons in terms of the light and heavy mass eigenstates $W_{1}$ and $W_{2}$ are given by $W_{L, R}^{+\mu}=\cos \xi W_{1,2}^{+\mu} \mp \sin \xi e^{\mp i \alpha} W_{2,1}^{+\mu}$, where $\tan \xi=$ $\lambda \sin (2 \beta)$ with $\tan \beta=v_{2} / v_{1}$ and $\lambda=M_{W_{1}}^{2} / M_{W_{2}}^{2}$.

Direct searches for the $W_{R}$ boson provide a bound on $M_{W_{R}} \simeq M_{W_{2}}>4.8 \mathrm{TeV}$ [36-39], which is stronger than the constraints in flavor-changing processes [40,41]. As $\xi$ is bounded above by $\lambda$, we immediately obtain that $\xi \simeq \lambda \sin (2 \beta)<2.8 \times 10^{-4} \sin (2 \beta)$. A direct, albeit weaker, bound can be achieved in superallowed nuclear $\beta$ decays [42-45], $\xi \cos \alpha \leq 1.25 \times 10^{-3}$. Thus the value of $\xi$ 
crucially depends on $\beta$. It turns out $[46,47]$ that if the LR symmetry is taken to be generalized parity $(\mathcal{P})$, $|\sin \alpha \tan (2 \beta)|<2 m_{b} / m_{t}$, with $m_{b}$ and $m_{t}$ being the bottom and top quark masses, respectively, and no such constraint exists when generalized charge conjugation $(\mathcal{C})$ is the LR symmetry. From the theoretical perspective, the vacuum stability and perturbativity require $M_{W_{R}} \gtrsim 6 \mathrm{TeV}$ [48] and $\tan \beta<0.5$ [see Eq. (34) in Ref. [47] ], respectively. Hereafter, we will work in the case of $\mathcal{C}$ and assume $M_{W_{R}}=7 \mathrm{TeV}$ and $\alpha=0$.

For purposes of illustration, we follow Ref. [31] and assume "type-II dominance" for neutrino masses [49]. In this scenario, $m_{N_{i}} \propto m_{\nu_{i}}$, one has $V_{L}=V_{R}^{*}$ [31]. Using the light neutrino mass difference from solar and atmospheric neutrinos [54], and fixing the mass of heaviest right-handed neutrino $m_{N_{\max }}$, which is assumed to be $500 \mathrm{GeV}$ satisfying the experimental searches [36-39,55,56], it is possible to obtain all the neutrino masses in terms of the lightest neutrino mass $m_{\nu_{\min }}$.

The effective Lagrangian below the electroweak scale is

$$
\begin{aligned}
\mathcal{L}_{\text {eff }}= & \frac{G_{F}^{2}}{\Lambda_{\beta \beta}}\left[C_{3 R}\left(\mathcal{O}_{3+}^{++}-\mathcal{O}_{3-}^{++}\right)\left(\bar{e} e^{c}-\bar{e} \gamma_{5} e^{c}\right)\right. \\
& +C_{3 L}\left(\mathcal{O}_{3+}^{++}+\mathcal{O}_{3-}^{++}\right)\left(\bar{e} e^{c}-\bar{e} \gamma_{5} e^{c}\right) \\
& \left.+C_{1} \mathcal{O}_{1+}^{++}\left(\bar{e} e^{c}-\bar{e} \gamma_{5} e^{c}\right)+C_{1}^{\prime} \mathcal{O}_{1+}^{++\prime}\left(\bar{e} e^{c}-\bar{e} \gamma_{5} e^{c}\right)\right] \\
& + \text { H.c. }
\end{aligned}
$$

where [35]

$$
\begin{aligned}
\mathcal{O}_{3 \pm}^{++} & =\left(\bar{q}_{L}^{\alpha} \tau^{+} \gamma^{\mu} q_{L}^{\alpha}\right)\left(\bar{q}_{L}^{\beta} \tau^{+} \gamma_{\mu} q_{L}^{\beta}\right) \pm(L \rightarrow R) \\
\mathcal{O}_{1+}^{++} & =\left(\bar{q}_{L}^{\alpha} \tau^{+} \gamma^{\mu} q_{L}^{\alpha}\right)\left(\bar{q}_{R}^{\beta} \tau^{+} \gamma_{\mu} q_{R}^{\beta}\right), \\
\mathcal{O}_{1+}^{++^{\prime}} & =\left(\bar{q}_{L}^{\alpha} \tau^{+} \gamma^{\mu} q_{L}^{\beta}\right)\left(\bar{q}_{R}^{\beta} \tau^{+} \gamma_{\mu} q_{R}^{\alpha}\right),
\end{aligned}
$$

and $\alpha, \beta$ are the color indices, $\tau^{ \pm}=\left(\tau^{1} \pm i \tau^{2}\right) / 2, \tau^{1}$ and $\tau^{2}$ are the Pauli matrices.

Wilson coefficients $C_{3 R}, C_{3 L}$, and $C_{1}$ are obtained by integrating out the $W_{1,2}$ and $N_{i}$ arising, respectively, from the amplitudes in Figs. 1(a), 1(c), and 1(d). We evolve them from the scale $\mu=M_{W_{2}}$ to an appropriately chosen hadronic scale $\Lambda_{H}=2 \mathrm{GeV}$ [57]. The renormalization group evolution (RGE) proceeds in two steps: (a) $\mu=M_{W_{2}} \rightarrow M_{W_{1}}$ and (b) $\mu=M_{W_{1}} \rightarrow \Lambda_{H}$ and it gives [50,58,59]

$$
\begin{aligned}
\left(\begin{array}{l}
C_{1}\left(\Lambda_{H}\right) \\
C_{1}^{\prime}\left(\Lambda_{H}\right)
\end{array}\right) & =\left(\begin{array}{cc}
0.90 & 0 \\
0.48 & 2.32
\end{array}\right)\left(\begin{array}{l}
C_{1}\left(M_{W_{1}}\right) \\
C_{1}^{\prime}\left(M_{W_{1}}\right)
\end{array}\right), \\
C_{3 L}\left(\Lambda_{H}\right) & =0.81 C_{3 L}\left(M_{W_{1}}\right), \\
C_{3 R}\left(\Lambda_{H}\right) & =0.71 C_{3 R}\left(M_{W_{2}}\right),
\end{aligned}
$$

where $C_{1}^{\prime}\left(M_{W_{1}}\right)=0$ and it appears due to the RGE of $C_{1}$. In Eq. (6), the nonvanishing Wilson coefficients at the electroweak scale are given by $C_{1}\left(M_{W_{1}}\right)=-4 \lambda \xi$, $C_{3 L}\left(M_{W_{1}}\right)=\xi^{2}$, and $C_{3 R}\left(M_{W_{2}}\right)=\lambda^{2}\left(1+4 \Lambda_{\beta \beta}^{2} / M_{\Delta_{R}}^{2}\right)$, with $1 / \Lambda_{\beta \beta}=\sum_{i=1}^{3}\left|V_{R e i}\right|^{2} / m_{N_{i}}$. Note that $\mathcal{O}_{3 L} \equiv \mathcal{O}_{3+}^{++}+$ $\mathcal{O}_{3-}^{++}$and $\mathcal{O}_{1+}^{++}$are matched to effective operators above the electroweak scale, which however do not evolve under QCD running [50], so that the RGE only includes step (b).

The doubly charged scalar, depicted in Fig. 1(b), contributes solely to $C_{3 R}$. When the LR symmetry holds, this contribution is negligible due to collider bounds [60] and charged lepton flavor violation constraints [31]. On the contrary, when the LR symmetry is explicitly broken, these constraints are relaxed and the corresponding contribution to the $0 \nu \beta \beta$ decay rate can be appreciable. For a discussion, and the possible interplay with prospective future low- and highenergy probes, see Ref. [61]. Here, we assume a LR-symmetric Lagrangian and leave the analysis of the interesting case when it is broken for a future work.

We now map the operators in Eq. (2) at GeV scale $\sim \Lambda_{H}$ onto an effective hadron-lepton Lagrangian below that scale $[35,50,62]$ using chiral perturbation theory $(\chi \mathrm{PT})[63,64]$. Matching entails identifying all operators at a given chiral order that transform under chiral SU(2) the same way as the four-quark factor of a given operator in Eq. (2) $[35,65]$. We refer the reader to Ref. [35] for a detailed derivation, and here simply quote the results. For another approach in which quark bilinears are mapped onto nucleon matrix elements, one can refer to Refs. [66-68] for generic studies of $0 \nu \beta \beta$ decay and Ref. [69] in the context of left-right symmetric models.

The hadron-lepton Lagrangian for the $\pi \pi \bar{e} e^{c}, \bar{N} N \pi \bar{e} e^{c}$, and $\bar{N} N \bar{N} N \bar{e} e^{c}$ operators up to next-to-next-to-leading order (NNLO) in chiral expansion is [35]

$$
\begin{aligned}
\mathcal{L}_{\chi \mathrm{PT}}= & \frac{G_{F}^{2} F_{\pi}^{2}}{\Lambda_{\beta \beta}}\left\{\Lambda_{\chi}^{2} \pi^{-} \pi^{-} \bar{e}\left(\beta_{1}+\beta_{2} \gamma^{5}\right) e^{c}\right. \\
& +\partial_{\mu} \pi^{-} \partial^{\mu} \pi^{-} \bar{e}\left(\beta_{3}+\beta_{4} \gamma^{5}\right) e^{c} \\
& +\Lambda_{\chi} / F_{\pi} \bar{N} i \gamma_{5} \tau^{+} \pi^{-} N \bar{e}\left(\zeta_{5}+\zeta_{6} \gamma^{5}\right) e^{c} \\
& +1 / F_{\pi}^{2} \bar{N} \tau^{+} N \bar{N} \tau^{+} N \bar{e}\left(\xi_{1}+\xi_{4} \gamma_{5}\right) e^{c} \\
& + \text { H.c. }\} .
\end{aligned}
$$

The first two-pion term contributes to the amplitude $\mathcal{A}\left(n n \rightarrow p p e^{-} e^{-}\right)$at order of $p^{-2}$ with $p \lesssim m_{\pi}$ being the typical momentum transfer. When this leading-order (LO) amplitude $\mathcal{A}^{\mathrm{LO}}$ is present as in the minimal LRSM, it can give a dominant long-range contribution to the half-life of $0 \nu \beta \beta$ decay [35]. The one-pion and fournucleon and another two-pion terms, however, contribute 
at next-to-next-to $\mathrm{LO}$ to the amplitude $\mathcal{A}^{\mathrm{NNLO}} \sim p^{0}$. The dimensionless coefficients are expressed as [35] $\beta_{1}=-\beta_{2}=\ell_{1}^{\pi \pi} C_{1}+\ell_{1}^{\pi \pi \prime} C_{1}^{\prime}, \beta_{3}=-\beta_{4}=\ell_{3}^{\pi \pi}\left(C_{3 L}+C_{3 R}\right)$, $\zeta_{5}=-\zeta_{6}=\ell_{3}^{\pi N}\left(C_{3 L}+C_{3 R}\right)$, and $\xi_{1}=-\xi_{4}=\ell_{1}^{N N} C_{1}+$ $\ell_{1}^{N N \prime} C_{1}^{\prime}+\ell_{3}^{N N}\left(C_{3 L}+C_{3 R}\right)$. Furthermore, $g_{A}=1.271$, $\Lambda_{\chi}=4 \pi F_{\pi}$, with $F_{\pi}=92.28 \mathrm{MeV}$, and $\ell_{i}$ are the lowenergy constants (LECs). Using the lattice calculations [70], we get $\ell_{1}^{\pi \pi}=-(0.71 \pm 0.07), \ell_{1}^{\pi \pi \prime}=-(2.98 \pm 0.22)$, and $\ell_{3}^{\pi \pi}=0.60 \pm 0.03$ in the modified minimal substraction $(\overline{\mathrm{MS}})$ scheme at $\mu=2 \mathrm{GeV}$ [50]. The LECs for $\bar{N} N \pi \bar{e} e^{c}$ and $\bar{N} N \bar{N} N \bar{e} e^{c}$ interactions are unknown and are estimated using the naive dimensional analysis [71] with $\ell_{3}^{\pi N} \sim \mathcal{O}(1)$ and $\ell_{1}^{N N}, \ell_{1}^{N N \prime}, \ell_{3}^{N N} \sim \mathcal{O}(1)$.

The four-nucleon interaction in Eq. (7) merits a more detailed discussion. In Ref. [50], it was observed that a consistent renormalization of the amplitude induced by the operators $\mathcal{O}_{1+}^{++}, \mathcal{O}_{1+}^{++\prime}$ requires inclusion of a LO fournucleon counterterm [72]. While its presence does not change the magnitude of the NNLO contributions (barring accidental cancellations), it does introduce additional hadronic uncertainties at LO. To check how this new source of uncertainty might affect our results, we have taken the natural assumption that this new contribution gives an additional $100 \%$ contribution to the decay rate and found that our conclusions remain the same. Finally and notwithstanding the above arguments, the uncertainty might be bigger, as suggested by the RGE analysis of Ref. [50]. However, this issue is still far from settled until the finite piece of the $\mathrm{LO}$ four-nucleon counterterm is taken from a more reliable source, such as lattice QCD, for instance.

From Eq. (7), we obtain the decay half-life,

$$
\begin{aligned}
\left(T_{1 / 2}^{0 \nu}\right)^{-1} & =G_{0 \nu} \mathcal{M}_{\nu}^{2}\left|m_{\beta \beta}\right|^{2} \\
& =G_{0 \nu} \mathcal{M}_{\nu}^{2}\left(\left|m_{\nu}^{e e}\right|^{2}+\left|m_{N}^{e e}\right|^{2}\right),
\end{aligned}
$$

where

$$
m_{\nu}^{e e} \simeq \sum_{i=1}^{3}\left|V_{L e i}\right|^{2} m_{\nu_{i}}\left(1+\ell_{\nu}^{N N} \delta_{N N}^{\nu}\right)
$$

and

$$
\begin{aligned}
\left|m_{N}^{e e}\right|^{2}= & \frac{\Lambda_{\chi}^{4}}{72 \Lambda_{\beta \beta}^{2}} \frac{\mathcal{M}_{0}^{2}}{\mathcal{M}_{\nu}^{2}} \times\left[\left(\beta_{1}-\zeta_{5} \delta_{N \pi}-\beta_{3} \delta_{\pi \pi}+\xi_{1} \delta_{N N}\right)^{2}\right. \\
& \left.+\left(\beta_{2}-\zeta_{6} \delta_{N \pi}-\beta_{4} \delta_{\pi \pi}+\xi_{4} \delta_{N N}\right)^{2}\right],
\end{aligned}
$$

with $m_{N}=939 \mathrm{MeV}$ and

$$
\begin{gathered}
\delta_{\pi \pi}=\frac{2 m_{\pi}^{2}}{\Lambda_{\chi}^{2}} \frac{\mathcal{M}_{2}}{\mathcal{M}_{0}}, \quad \delta_{N \pi}=\frac{\sqrt{2} m_{\pi}^{2}}{g_{A} \Lambda_{\chi} m_{N}} \frac{\mathcal{M}_{1}}{\mathcal{M}_{0}}, \\
\delta_{N N}^{\nu}=\frac{2 m_{\pi}^{2}}{g_{A}^{2} \Lambda_{\chi}^{2}} \frac{\mathcal{M}_{N N}}{\mathcal{M}_{\nu}},
\end{gathered}
$$

Future ton-scale experiments searching for $0 \nu \beta \beta$ decay in ${ }^{136} \mathrm{Xe}$ are considered for numerical results. The phase

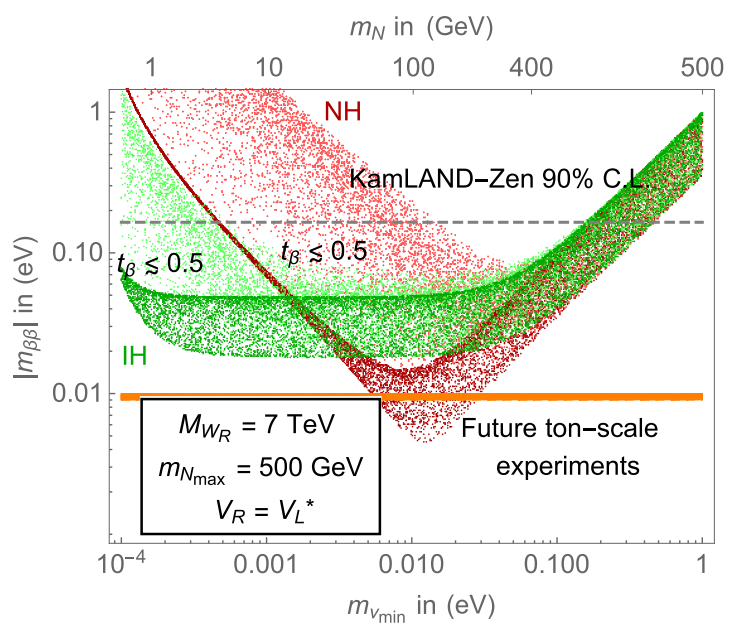

FIG. 2. Effective Majorana mass as a function of the lightest neutrino mass. The central values of the mixing angles and the Dirac $C P$-violating phase in $V_{L}$ are quoted from Ref. [54], and the Majorana phases are marginalized. The allowed regions with $\tan \beta=0$ and $\tan \beta \leq 0.5$ are depicted in darker and lighter colors. Red (green) dots denote the $\mathrm{NH}$ (IH) of neutrino mass ordering. Gray and orange lines represent the current and expected limits from the KamLAND-Zen Collaboration [7] and future ton-scale experiment $[9,10]$, respectively. The lightest heavy neutrino mass is also given in the upper horizontal axis.

1space factor $G_{0 \nu}^{-1}=7.11 \times 10^{24} \mathrm{eV}^{2} \mathrm{yr}[73,74]$, and the nuclear matrix elements $\mathcal{M}_{\nu}=2.91, \mathcal{M}_{0}=-2.64$, $\mathcal{M}_{1}=-5.52$ and $\mathcal{M}_{2}=-4.20, \mathcal{M}_{N N}=-1.53$ are quoted [75]. We obtain that $\delta_{\pi \pi}=0.046, \quad \delta_{N N \pi}=0.042$, $\delta_{N N}^{\nu}=-0.0096$, and $\delta_{N N}=0.063$, clearly showing the expected chiral suppression $\left|\mathcal{A}^{\mathrm{NNLO}} / \mathcal{A}^{\mathrm{LO}}\right| \sim 15-20$ or even larger. Again, the LEC $\ell_{\nu}^{N N} \sim \mathcal{O}(1)$ in naive dimensional analysis and is larger, requiring LO $\bar{N} N \bar{N} N \bar{e} e^{c}$ counterterm [72].

In Fig. 2, we show the effective Majorana mass $\left|m_{\beta \beta}\right|$ as a function of $m_{\nu_{\min }}$ with $m_{N_{\max }}=500 \mathrm{GeV}$ and $M_{W_{R}}=7 \mathrm{TeV}$. To illustrate the impact of the LR contribution, we give the allowed regions with $\tan \beta=0$ (studied in Refs. [31,76]) and $0<\tan \beta \leq 0.5$ in darker and lighter colors, respectively. For most of the $\tan \beta>0$ parameter space, the long-range pion exchange contribution dominates over other contributions. In Fig. 3, we plot the $\left|m_{\beta \beta}\right|$ as a function of $\sum m_{\nu}$ along with the current upper bound from cosmology experiments [15]. In particular, we see from Fig. 3 (upper panel) that in the NH, inclusion of the long-range contribution opens up a significant portion of parameter space accessible to ton-scale experiments. Thus, even if the future cosmic microwave background and large scale structure data would exclude the IH [16], there are good prospects of new physics at the $\mathrm{TeV}$ scale giving the dominant contribution to the $0 \nu \beta \beta$ decay rate in future ton-scale experiments. 

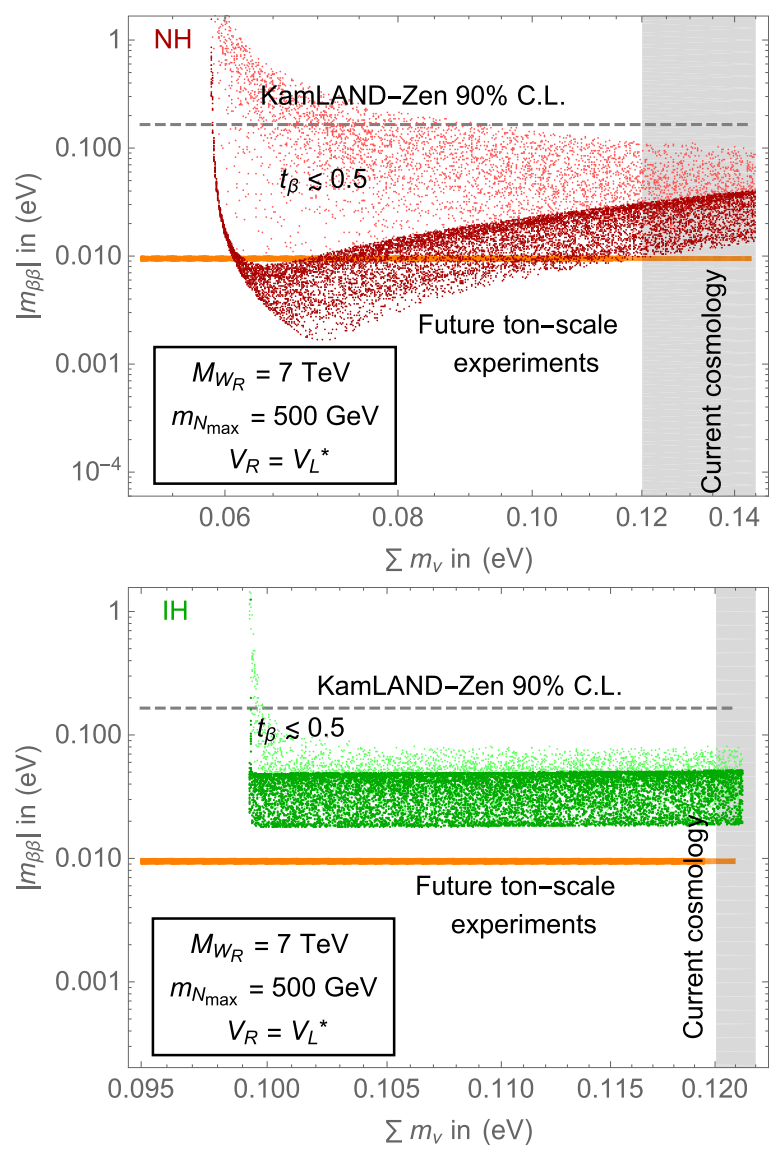

FIG. 3. Effective Majorana mass as a function of the sum of light neutrino masses. The red and green regions as well as gray and the orange lines have the same meaning as in Fig. 2. The current constraint from cosmological experiments [15] is depicted in the gray region.

G. L. and J. C. V. would like to thank Jordy de Vries for many valuable discussions. G. L. thanks Wouter Dekens and Jordy de Vries for fruitful discussions on the half-life calculations regarding Refs. [50,77], and Xiao-Dong Ma and Jiang-Hao Yu for the discussions on RGEs. J. C. V. was supported in part under the U.S. Department of Energy Contract No. DE-SC0015376. G. L. and M. R.-M. were supported in part under U.S. Department of Energy Contract No. DE-SC0011095. M. R.-M. was also supported in part under National Science Foundation of China Grant No. 19Z103010239.

*Corresponding author.

ligang@umass.edu

†mjrm@sjtu.edu.cn; mjrm@physics.umass.edu

*jvasquezcarm@umass.edu

[1] M. Tanabashi et al. (Particle Data Group), Phys. Rev. D 98, 030001 (2018).

[2] F. Capozzi, E. Di Valentino, E. Lisi, A. Marrone, A. Melchiorri, and A. Palazzo, Phys. Rev. D 101, 116013 (2020).
[3] F. Capozzi, E. Di Valentino, E. Lisi, A. Marrone, A. Melchiorri, and A. Palazzo, Phys. Rev. D 95, 096014 (2017).

[4] M. Fukugita and T. Yanagida, Phys. Lett. B 174, 45 (1986).

[5] W. Furry, Phys. Rev. 56, 1184 (1939).

[6] J. Schechter and J. W. F. Valle, Phys. Rev. D 25, 2951 (1982).

[7] A. Gando et al. (KamLAND-Zen Collaboration), Phys. Rev. Lett. 117, 082503 (2016); 117, 109903(E) (2016).

[8] G. L. Fogli, E. Lisi, A. Marrone, and A. Palazzo, Prog. Part. Nucl. Phys. 57, 742 (2006).

[9] J. B. Albert et al. (nEXO Collaboration), Phys. Rev. C 97, 065503 (2018).

[10] S. A. Kharusi et al. (nEXO Collaboration), arXiv:1805.11142.

[11] N. Abgrall et al. (LEGEND Collaboration), AIP Conf. Proc. 1894, 020027 (2017).

[12] E. Armengaud et al., Eur. Phys. J. C 80, 44 (2020).

[13] W. Armstrong et al. (CUPID Collaboration), arXiv:1907.09376.

[14] J. Paton (SNO+Collaboration), in Prospects in Neutrino Physics (2019).

[15] N. Aghanim et al. (Planck Collaboration), Astron. Astrophys. 641, A6 (2020).

[16] T. Brinckmann, D. C. Hooper, M. Archidiacono, J. Lesgourgues, and T. Sprenger, J. Cosmol. Astropart. Phys. 01 (2019) 059.

[17] K. Abazajian et al., arXiv:1907.04473.

[18] M. Hazumi et al., Proc. SPIE Int. Soc. Opt. Eng. 8442, 844219 (2012).

[19] M. E. Levi et al. (DESI Collaborations), arXiv:1907.10688.

[20] R. Scaramella et al. (Euclid Collaboration), IAU Symp. 10, 375 (2014).

[21] J. C. Pati and A. Salam, Phys. Rev. D 10, 275 (1974); 11, 703(E) (1975).

[22] R. Mohapatra and J.C. Pati, Phys. Rev. D 11, 2558 (1975).

[23] G. Senjanovic and R. N. Mohapatra, Phys. Rev. D 12, 1502 (1975).

[24] G. Senjanović, Nucl. Phys. B153, 334 (1979).

[25] R. N. Mohapatra and G. Senjanovic, Phys. Rev. Lett. 44, 912 (1980).

[26] R. N. Mohapatra and G. Senjanovic, Phys. Rev. D 23, 165 (1981).

[27] R. Mohapatra, Nucl. Phys. B, Proc. Suppl. 77, 376 (1999).

[28] G. Prezeau, Phys. Lett. B 633, 93 (2006).

[29] V. Cirigliano, A. Kurylov, M. J. Ramsey-Musolf, and P. Vogel, Phys. Rev. Lett. 93, 231802 (2004).

[30] W. Rodejohann, Int. J. Mod. Phys. E 20, 1833 (2011).

[31] V. Tello, M. Nemevsek, F. Nesti, G. Senjanovic, and F. Vissani, Phys. Rev. Lett. 106, 151801 (2011).

[32] P. S. Bhupal Dev, S. Goswami, and M. Mitra, Phys. Rev. D 91, 113004 (2015).

[33] J. Chakrabortty, H. Z. Devi, S. Goswami, and S. Patra, J. High Energy Phys. 08 (2012) 008.

[34] J. Barry and W. Rodejohann, J. High Energy Phys. 09 (2013) 153.

[35] G. Prezeau, M. Ramsey-Musolf, and P. Vogel, Phys. Rev. D 68, 034016 (2003).

[36] M. Aaboud et al. (ATLAS Collaboration), Phys. Lett. B 798, 134942 (2019).

[37] M. Aaboud et al. (ATLAS Collaboration), J. High Energy Phys. 01 (2019) 016. 
[38] A. M. Sirunyan et al. (CMS Collaboration), J. High Energy Phys. 05 (2018) 148.

[39] A. M. Sirunyan et al. (CMS Collaboration), J. High Energy Phys. 07 (2017) 121.

[40] S. Bertolini, A. Maiezza, and F. Nesti, Phys. Rev. D 89, 095028 (2014).

[41] Y. Zhang, H. An, X. Ji, and R. N. Mohapatra, Nucl. Phys. B802, 247 (2008).

[42] C.-Y. Seng, M. Gorchtein, H. H. Patel, and M. J. RamseyMusolf, Phys. Rev. Lett. 121, 241804 (2018).

[43] P. Zyla et al. (Particle Data Group), Prog. Theor. Exp. Phys. 2020, 083C01 (2020).

[44] V. Cirigliano, S. Gardner, and B. Holstein, Prog. Part. Nucl. Phys. 71, 93 (2013).

[45] M. González-Alonso, O. Naviliat-Cuncic, and N. Severijns, Prog. Part. Nucl. Phys. 104, 165 (2019).

[46] G. Senjanović and V. Tello, Phys. Rev. Lett. 114, 071801 (2015).

[47] G. Senjanović and V. Tello, Phys. Rev. D 94, 095023 (2016).

[48] A. Maiezza, G. Senjanović, and J. C. Vasquez, Phys. Rev. D 95, 095004 (2017).

[49] The type-I seesaw scenario was studied in Ref. [50] where the new physics contribution can also dominate over the standard light neutrino exchange scenario. For the studies of $0 \nu \beta \beta$ decay in other variants of left-right symmetric models, see, for example, Refs. [51-53].

[50] V. Cirigliano, W. Dekens, J. de Vries, M. L. Graesser, and E. Mereghetti, J. High Energy Phys. 12 (2018) 097.

[51] P.-H. Gu, J. High Energy Phys. 09 (2016) 152.

[52] C. Majumdar, S. Patra, S. Senapati, and U. A. Yajnik, Nucl. Phys. B951, 114875 (2020).

[53] S. Senapati, S. Patra, P. Pritimita, and C. Majumdar, Nucl. Phys. B954, 115000 (2020).

[54] P. de Salas, D. Forero, S. Gariazzo, P. Martínez-Miravé, O. Mena, C. Ternes, M. Tórtola, and J. Valle, J. High Energy Phys. 02 (2021) 071.

[55] A. M. Sirunyan et al. (CMS Collaboration), J. High Energy Phys. 01 (2019) 122.

[56] G. Aad et al. (ATLAS Collaboration), J. High Energy Phys. 10 (2019) 265.
[57] S. Aoki et al. (Flavour Lattice Averaging Group), Eur. Phys. J. C 80, 113 (2020).

[58] V. Cirigliano, W. Dekens, J. de Vries, M. Graesser, and E. Mereghetti, J. High Energy Phys. 12 (2017) 082.

[59] Y. Liao, X.-D. Ma, and H.-L. Wang, J. High Energy Phys. 01 (2020) 127.

[60] M. Aaboud et al. (ATLAS Collaboration), Eur. Phys. J. C 78, 199 (2018).

[61] P. S. Bhupal Dev, M. J. Ramsey-Musolf, and Y. Zhang, Phys. Rev. D 98, 055013 (2018).

[62] M. L. Graesser, J. High Energy Phys. 08 (2017) 099.

[63] V. Bernard, N. Kaiser, and U.-G. Meissner, Int. J. Mod. Phys. E 04, 193 (1995).

[64] J. Gasser and H. Leutwyler, Ann. Phys. (N.Y.) 158, 142 (1984).

[65] D. B. Kaplan and M. J. Savage, Nucl. Phys. A556, 653 (1993); A570, 833(E) (1994); A580, 679(E) (1994).

[66] M. Doi, T. Kotani, and E. Takasugi, Prog. Theor. Phys. Suppl. 83, 1 (1985).

[67] H. Pas, M. Hirsch, H. V. Klapdor-Kleingrothaus, and S. G. Kovalenko, Phys. Lett. B 498, 35 (2001).

[68] L. Graf, F. F. Deppisch, F. Iachello, and J. Kotila, Phys. Rev. D 98, 095023 (2018).

[69] M. Hirsch, H. V. Klapdor-Kleingrothaus, and O. Panella, Phys. Lett. B 374, 7 (1996).

[70] A. Nicholson et al., Phys. Rev. Lett. 121, 172501 (2018).

[71] A. Manohar and H. Georgi, Nucl. Phys. B234, 189 (1984).

[72] V. Cirigliano, W. Dekens, J. De Vries, M. L. Graesser, E. Mereghetti, S. Pastore, and U. Van Kolck, Phys. Rev. Lett. 120, 202001 (2018).

[73] J. Kotila and F. Iachello, Phys. Rev. C 85, 034316 (2012).

[74] S. Stoica and M. Mirea, Phys. Rev. C 88, 037303 (2013).

[75] J. Hyvarinen and J. Suhonen, Phys. Rev. C 91, 024613 (2015).

[76] S.-F. Ge, M. Lindner, and S. Patra, J. High Energy Phys. 10 (2015) 077.

[77] W. Dekens, J. de Vries, K. Fuyuto, E. Mereghetti, and G. Zhou, J. High Energy Phys. 06 (2020) 097. 